\title{
CALCULATION OF THE IMPROPER INTEGRALS FOR FOURIER BOUNDARY ELEMENT METHOD
}

\author{
Edyta Lukasik $^{1}$, Beata Pańczyk ${ }^{1}$, Jan Sikora ${ }^{2}$ \\ ${ }^{1}$ Lublin University of Technology, Electrical Engineering and Computer Science Faculty, Institute of Computer Science \\ ${ }^{2}$ Lublin University of Technology, Electrical Engineering and Computer Science Faculty, Institute of Electronics and Information Technology
}

Abstract. The traditional Boundary Element Method (BEM) is a collection of numerical techniques for solving some partial differential equations. The classical BEM produces a fully populated coefficients matrix. With Galerkin Boundary Element Method (GBEM) is possible to produce a symmetric coefficients matrix. The Fourier BEM is a more general numerical approach. To calculate the final matrix coefficients it is necessary to find the improper integrals. The article presents the method for calculation of such integrals.

Keywords: Fourier Boundary Element Method, numerical integration, improper integrals

\section{WYZNACZANIE CALEK NIEWLAŚCIWYCH W METODZIE ELEMENTÓW BRZEGOWYCH FOURIERA}

Streszczenie. Tradycyjna metoda elementów brzegowych(MEB) prowadzi wefekcie do rozwiązania układu równań liniowych z petna macierza współczynników. Stosujac podejście Galerkina ostateczny układ równań liniowych jest reprezentowany macierza symetryczna. W podejściu Fouriera, wspótczynniki układu równań wyznaczane sa w przestrzeni Fouriera co pozwala uniknać problemów z całkowaniem całek nieosobliwych, ale powoduje konieczność obliczania całek niewłaściwych. W artykule zaprezentowano algorytm obliczania takich całek.

Słowa kluczowe: metoda elementów brzegowych Fouriera, całkowanie numeryczne, całki niewłaściwe

\section{Introduction}

Basic integral equation for the Boundary Element Method (BEM) is constructed by the convolution with the fundamental solution [2,3,6]. Figure 1 presents domain $\Omega \subset R^{n}$ with Dirichlet and Neumann boundary.

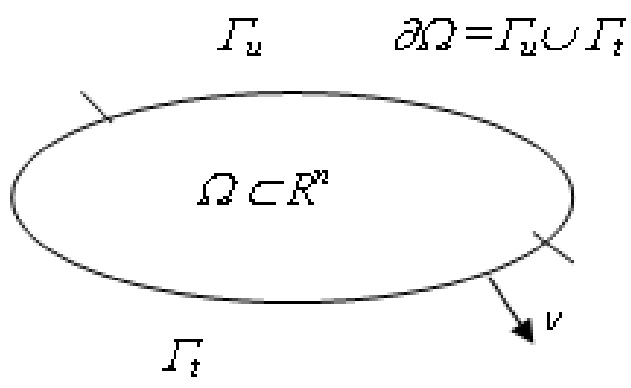

Fig. 1. The domain $\Omega$

The basic principles of the traditional BEM are presented for the paradigmatic example of the $n$-dimensional stationary heat conduction described by [2]:

$$
\begin{gathered}
\Delta u(x)=-f(x), x \in \Omega \subset R^{n}, \Delta=\sum_{k=1}^{n} \partial^{2} / \partial x_{k}^{2} \\
u(x)=u_{\Gamma}(x), \quad x \in \Gamma_{u} \subset \Omega, \\
t(x)=t_{\Gamma}(x), \quad x \in \Gamma_{t} \subset \Omega .
\end{gathered}
$$

where: $\Delta$ - Laplace operator, $u$ - the unknown quantity, $f$ - the known volume sources in $\Omega$.

The flux on the boundary is:

$$
t=\mathrm{A}_{t} u=-\partial_{v} u=-v \cdot \nabla u,
$$

where:

$\nabla, v$ - the gradient and the outer unit normal,

$A_{t}=-v \cdot \nabla$ - the boundary operator,

$\partial / \partial x_{k}$ - the partial derivatives denotes $\partial_{k}$,

$\boldsymbol{x}$ - $n$-dimensional vector,

$d \boldsymbol{x}$ - the short form for $d x_{1} d x_{2}$ (or $d x_{1} d x_{2} d x_{3}$ ).

To obtain a well posed problem, half of the boundary data (either $u$ on $\Gamma_{u}$ or $t$ on $\Gamma_{t}$ ) should be defined by boundary conditions, i.e. $\Gamma_{u} \cup \Gamma_{t}=\partial \Omega$.

\section{The distribution theory}

Distributions are objects which generalize functions [2, 8]. They extend the concept of derivative to all locally integral functions and are used to formulate generalized solutions of partial differential equations. They are important in physics and engineering where many non-continuous problems naturally lead to differential equations whose solutions or initial conditions are distributions, such as the Dirac delta distribution.

The basic idea is to identify functions with abstract linear functionals on a space of well-behaved test functions.

For example, let: $u: R \rightarrow R$, be a locally integrable, and $\phi: R \rightarrow R$,be a smooth (infinitely differentiable) function with compact support (i.e., identically zero outside of some bounded set). The function $\phi$ is the test function and:

$$
u(\phi)=\langle u, \phi\rangle=\int_{R} u \phi d x<\infty .
$$

This is a real number which depends on $\phi$. The function $u$ is then a continuous linear functional on the space which consists of all the test functions $\phi$. The set of generalized functions $u$ include all linear and continuous functionals. They are defined by some test functions $\phi$. Properties of the test functions define the set of generalized functions.

The test functions: $\phi(x) \in D(\Omega)=C_{0}^{\infty}(\Omega)$, are bounded, posses a compact support and are infinitely continuously differentiable. They and all their derivatives vanish at the boundary.

Distribution $u \in D^{\prime}(\Omega)$ is defined by the scalar product with the test function $\phi$ :

$$
u(\phi)=\langle u, \phi\rangle=\int_{R^{n}} u(x) \overline{\phi(x)} d x<\infty .
$$

The differentiation of generalized functions is defined as:

$$
\left\langle\partial_{k} u, \phi\right\rangle=-\left\langle u, \partial_{k} \phi\right\rangle, \phi \in C_{0}^{\infty} .
$$

Because of the definition of the test function $\phi$, distributions are infinitely differentiable. Jumps and singularities can be differentiated [2].

By using a larger space of test functions, it is possible to define the tempered distributions, useful for the Fourier transformation in generality. All tempered distributions have a Fourier transformation, but not all distributions have one [2].

The invariance of the scalar product concerning the Fourier transformation is called Parseval's identity:

$$
\left\langle u_{1}, u_{2}\right\rangle=\frac{1}{(2 \pi)^{n}}\left\langle\hat{u}_{1}, \hat{u}_{2}\right\rangle \text {. }
$$

It is possible to define the Fourier transformation of tempered distributions. These include all the integrable functions, as well as well-behaved functions of polynomial growth and distributions of compact support, and have the added advantage that the Fourier transformation of any tempered distribution is again a tempered distribution. 


\section{The special distributions}

The $n$-dimensional Dirac distribution:

$$
\delta(x)=\prod_{k=1}^{n} \delta\left(x_{k}\right)
$$

is defined by:

$$
\begin{aligned}
& \int_{R^{n}} \delta(x) d x=1, x \in R^{n} ; \\
& \delta(x)=0 \text { for all }|\mathrm{x}| \neq 0 .
\end{aligned}
$$

The Dirac distribution is the identity object concerning convolution:

$$
u=u * \delta=\int_{R^{n}} u(y) \delta(x-y) d y, x, y \in R^{n},
$$

and its Fourier transformation is:

$$
\delta(x) \stackrel{F}{\longleftrightarrow} 1, x \in R^{n} .
$$

The Heaviside distribution is obtained by the integration of the Dirac distribution:

$$
H(x)=\int_{-\infty}^{x} \delta(y) d y=\left\{\begin{array}{ll}
1 & x>0 \\
0 & x<0
\end{array} .\right.
$$

In the literature, there are several definitions for the value at $x=0$. For the linear distribution it is determined by:

$$
\langle H(x), \delta(x)\rangle=\kappa=\frac{1}{2}, x \in R^{1} .
$$

For the multidimensional Heaviside distribution, the cutoff distribution for a domain $\Omega \in R^{n}$ is defined:

$$
\chi(x):=\left\{\begin{array}{cc}
1 & x \in \Omega \\
\kappa(x) & x \in \partial \Omega \\
0 & x \notin \bar{\Omega}=\Omega \cup \partial \Omega
\end{array},\right.
$$

which can be expressed by:

$$
\chi(x)=H(\psi(x)),
$$

with a function $\psi \in C^{\infty}\left(R^{n}\right)$

The integration of a distribution $u$ over the domain $\Omega$ can be described by:

$$
\langle H(\psi), u\rangle=\int_{\psi \geq 0} u(x) d x .
$$

The main advantage of the theory of distribution is that it re-establishes differentiation as the simple procedure and all quantities are differentiable even if they exhibit singularities and jumps [2].

\section{Fourier BEM}

To obtain the Fourier transformation of the Boundary Integral Equations (BIE), all quantities have to be extended from $\Omega$ to $R_{n}$. It can be done by defining a cutoff distribution $\chi$ [2]. All quantities are multiplied by $\chi$ and finally transformed into Fourier space. Mathematically this extension and transformation is justified only in the frame of the theory of distributions [2, 8].

The main advantage of the distributional BIE is that the integrals extend formally over the entire $R_{n}$ and therefore the Fourier transformation can be applied to these integral equation.

For the definition of the trial functions it is needed to define a cutoff distribution [2] for a rectangular element:

$$
\begin{gathered}
\chi^{0}(x):=H\left(x_{1}\right) H\left(1-x_{1}\right) \delta\left(x_{2}\right), x \in R^{2}, \\
\chi^{0}(x):=H\left(x_{1}\right) H\left(1-x_{1}\right) H\left(x_{2}\right) H\left(1-x_{2}\right) \delta\left(x_{3}\right), x \in R^{3} .
\end{gathered}
$$

The trial functions are obtained by multiplying $\chi^{0}(x)$ and $p^{0}(x) \in C^{\infty}\left(R^{n}\right)$ :

$$
\phi^{0}(x):=\chi^{0}(x) p^{0}(x) .
$$

The trial functions $\phi_{u, t}^{i}(x)$ on arbitrary straight elements are obtained by translation and/or dilation operators:

$$
\begin{aligned}
& T^{i}: \phi^{0} \rightarrow \phi_{u, t}^{i}=\phi^{0}\left(x-b^{i}\right), \\
& D^{i}: \phi^{0} \rightarrow \phi_{u, t}^{i}=\phi^{0}\left(a^{i} x\right),
\end{aligned}
$$

with the translation vector $\mathbf{b}_{i}$ and the dilation matrix $\mathbf{a}_{i}$.

Finally the unknown and the known quantities on the boundaries are approximated by:

$$
\delta(x) u(x) \approx \sum_{i}^{N_{u}} u^{i} \phi_{u}^{i}(x), \delta(\psi) \nabla \psi(x) \cdot \nabla u(x) \approx \sum_{i}^{N_{t}} t^{i} \phi_{t}^{i}(x) .
$$

The $n$-dimension Fourier transformation:

$$
F(u)=\hat{u}, u \in L_{1}\left(R^{n}\right), i=\sqrt{-1}
$$

is defined as:

$$
\hat{u}(\hat{x})=\int_{R^{n}} u(x) e^{-i<x, \hat{x}>} d x,<x, \hat{x}>=\sum_{k=1}^{n} x_{k} \hat{x}_{k} .
$$

The basics of Fourier BEM are two known theorems of the Fourier transformation.

The theorem of Parseval states the invariance of energy or work with respect to the dimensional Fourier transformation:

$$
\int_{R^{n}} \phi(x) u(x) d x=\frac{1}{(2 \pi)^{n}} \int_{R^{n}} \hat{\phi}(-\hat{x}) \hat{u}(\hat{x}) d \hat{x}, \quad x, \hat{x} \in R^{n}
$$

The convolution theorem links the convolution in the original space to a simple multiplication in the transformed space:

$$
\int_{R^{n}} \phi(y) u(x-y) d y \quad \stackrel{\mathrm{F}}{\longleftrightarrow} \hat{\phi}(\hat{x}) \hat{u}(\hat{x})
$$

In the notation:

$$
\begin{gathered}
\langle a, b\rangle=\int_{R^{n}} a(x) b(x) d x, \\
a * b=\int_{R^{n}} a(y) b(x-y) d y,
\end{gathered}
$$

these two theorems may be described as:

$$
\begin{gathered}
\langle\phi(x), u(x)\rangle=\frac{1}{(2 \pi)^{n}}\langle\hat{\phi}(-\hat{x}), \hat{u}(\hat{x})\rangle, \\
\phi(x) * u(x) \stackrel{\mathrm{F}}{\longleftrightarrow} \hat{\phi}(\hat{x}) \hat{u}(\hat{x}) .
\end{gathered}
$$

The Fourier BEM method analysed by [2] is especially of interest for cases where the fundamental solution is not known.

The transformation of the cutoff distribution $\chi_{0}$ is:

- for reference element in $R^{2}$ :

$$
\begin{aligned}
& \chi^{0}(x)=H\left(x_{1}\right) H\left(1-x_{1}\right) \delta\left(x_{2}\right) \\
& \stackrel{F}{\longleftrightarrow} \hat{\chi}^{0}(\hat{x})=\frac{i}{\hat{x}_{1}}\left(e^{-i \hat{x}_{1}}-1\right)
\end{aligned}
$$

- for reference element in $R^{3}$ :

$$
\begin{aligned}
& \chi^{0}(x)=H\left(x_{1}\right) H\left(1-x_{1}\right) H\left(x_{2}\right) H\left(1-x_{2}\right) \delta\left(x_{3}\right) \\
& \stackrel{F}{\longleftrightarrow} \hat{\chi}^{0}(\hat{x})=\frac{i}{\hat{x}_{1}}\left(e^{-i \hat{x}_{1}}-1\right) \frac{i}{\hat{x}_{2}}\left(e^{-i \hat{x}_{2}}-1\right)
\end{aligned}
$$

For straight elements and for arbitrary polynomial trial functions $p_{0}(x)$, the transformed expressions are analytically known in $R_{2}$ and $R_{3}$ [2].

The discretized Fourier BIE lead to an algebraic system identical to that obtained in the original space (Galerkin BEM [7]), where the matrices are computed in the transformed space and:

$$
\begin{array}{r}
F_{u}^{j}=\frac{1}{(2 \pi)^{n}}\left\langle\hat{\phi}_{t}^{j}(-\hat{x}), \hat{f}(\hat{x}) \hat{U}(\hat{x})\right\rangle, \\
H_{u}^{j i}=\frac{1}{(2 \pi)^{n}}\left\langle\hat{\phi}_{t}^{j}(-\hat{x}), \hat{\phi}_{t}^{i}(\hat{x}) \hat{U}(\hat{x})\right\rangle, \\
G_{u}^{j i}=\frac{1}{(2 \pi)^{n}}\left\langle\hat{\phi}_{t}^{j}(-\hat{x}), \hat{\phi}_{u}^{i}(\hat{x}) \hat{A}_{t}^{i} \hat{U}(\hat{x})\right\rangle, \\
K_{u}^{j i}:=\frac{1}{(2 \pi)^{n}}\left\langle\hat{\phi}_{t}^{j}(-\hat{x}), \hat{p}_{u}^{i}(\hat{x})\right\rangle .
\end{array}
$$




\section{Numerical example}

The problem of the numerical integration for Fourier BEM formulation is presented for the boundary integral equations limited to constant elements and 2D space. As the test example, the Dirichlet problem of the Poisson equation is considered [2].

The Dirichlet problem for Poisson equation:

$$
\begin{gathered}
\Delta u(x)=-f(x), x \in \Omega, \\
u(x)=u_{\Gamma}=0, x \in \Gamma,
\end{gathered}
$$

is solved in a quadratic two-dimensional domain $\Omega=[0,1] \times[0,1]$ at the boundaries $\mathrm{u}=0$. The interior is subjected to stationary heat source $f$. The boundary $\partial \Omega$ is divided into 8 elements (Fig. 2).

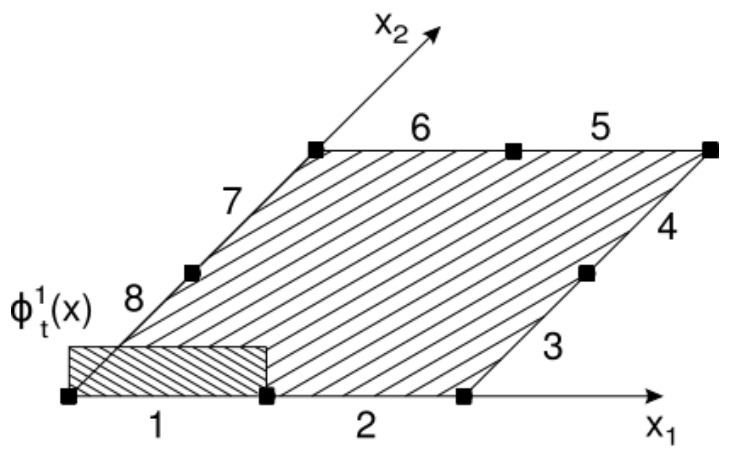

Fig. 2. Quadratic domain $\Omega$ with 8 boundary elements and constant trial function

The fundamental solution and its transformation for the Laplacian $\Delta$ are [2]:

$$
U=\frac{1}{2 \pi} \ln \sqrt{x_{1}^{2}+x_{2}^{2}} \stackrel{F}{\longleftrightarrow} \hat{U}=-\frac{1}{\hat{x}_{1}^{2}+\hat{x}_{2}^{2}}
$$

Taking into account the fact that $u=0$ at the boundaries, the general system of BIE can be reduced to:

$$
\begin{aligned}
& 0=\left\langle\phi_{t}^{j}, f_{\chi} * U\right\rangle+\sum_{i}^{N_{t}} t^{i}\left\langle\phi_{t}^{j}, \phi_{t}^{i} * U\right\rangle \\
& \stackrel{F}{\longleftrightarrow} 0=\left\langle\hat{\phi}_{t}^{j}(-\hat{x}), \hat{f}_{\chi} \hat{U}\right\rangle+\sum_{i}^{N_{t}} t^{i}\left\langle\hat{\phi}_{t}^{j}(-\hat{x}), \hat{\phi}_{t}^{i} \hat{U}\right\rangle
\end{aligned}
$$

For the $R^{2}$ elements the cuttoff distribution definition is described by eq.13 .

$$
\chi^{0}=H\left(x_{1}\right) H\left(1-x_{1}\right) \delta\left(x_{2}\right), \quad \mathrm{x} \in \mathrm{R}^{2},
$$

and its Fourier transformation is described by:

$$
\chi^{0}(x)=H\left(x_{1}\right) H\left(1-x_{1}\right) \delta\left(x_{2}\right) \stackrel{F}{\longleftrightarrow} \hat{\chi}^{0}(\hat{x})=\frac{i}{\hat{x}_{1}}\left(e^{-i \hat{x}_{1}}-1\right)
$$

In our example, for constant elements, the trial function and its Fourier transformation is:

$$
\phi^{0}=\chi^{0} \stackrel{F}{\longleftrightarrow} \hat{\phi}^{0}=\hat{\chi}^{0} .
$$

Additionally, the Fourier transformation for dilation and translation operators is described as (eq. 19):

$$
\begin{aligned}
& \phi^{0}(x-b) \stackrel{F}{\longleftrightarrow} \hat{\phi}^{0}(\hat{x}) e^{-i b \hat{x}}, \\
& \phi^{0}(a x) \stackrel{F}{\longleftrightarrow} \frac{1}{\mathrm{a}} \hat{\phi}^{0}(a \hat{x}) .
\end{aligned}
$$

The trial functions should be defined for 16 constant elements. For every element, the coefficients for Heaviside and Dirac distribution should be modified to receive the value of the product $H\left(x_{1}\right) H\left(1-x_{1}\right) \delta\left(x_{2}\right)$ equal to one inside the element and equal to zero outside. From the definition, the Dirac distribution is equal to one only for $\mathrm{x}=0$, and Heaviside distribution is equal to one for $\mathrm{x}>0$. For 8 elements we have the constant trial functions for the flux $\mathrm{t}[2]$ :

$$
\begin{aligned}
& \phi_{t}^{1}=H\left(x_{1}\right) H\left(1-2 x_{1}\right) \delta\left(x_{2}\right) \stackrel{F}{\longleftrightarrow} \\
& \hat{\phi}_{t}^{1}=\frac{i}{\hat{x}_{1} / 2}\left(e^{-i \hat{x}_{1} / 2}-1\right) \frac{1}{2}=i \frac{\left(e^{-i \hat{x}_{1} / 2}-1\right)}{\hat{x}_{1}},
\end{aligned}
$$

$$
\begin{aligned}
& \phi_{t}^{2}=H\left(2 x_{1}-1\right) H\left(1-x_{1}\right) \delta\left(x_{2}\right) \stackrel{F}{\longleftrightarrow} \\
& \hat{\phi}_{t}^{2}=\frac{1}{2} \frac{i}{\hat{x}_{1} / 2} e^{-i \hat{x}_{1} / 2}\left(e^{-i \hat{x}_{1} / 2}-1\right)=i \frac{\left(e^{-i \hat{x}_{1}}-e^{-i \hat{x}_{1} / 2}\right)}{\hat{x}_{1}} \cdot \\
& \phi_{t}^{3}=H\left(x_{2}\right) H\left(1-2 x_{2}\right) \delta\left(x_{1}-1\right) \quad \stackrel{F}{\longleftrightarrow} \\
& \hat{\phi}_{t}^{3}=\frac{i}{\hat{x}_{2} / 2}\left(e^{-i \hat{x}_{2} / 2}-1\right) \frac{1}{2} e^{-i \hat{x}_{1}}=i \frac{\left(e^{-i \hat{x}_{2} / 2}-1\right) e^{-i \hat{x}_{1}}}{\hat{x}_{2}} \\
& \phi_{t}^{4}=H\left(2 x_{2}-1\right) H\left(1-x_{2}\right) \delta\left(x_{1}-1\right) \stackrel{F}{\longleftrightarrow} \\
& \hat{\phi}_{t}^{4}=\frac{1}{2} \frac{i}{\hat{x}_{2} / 2} e^{-i \hat{x}_{2} / 2}\left(e^{-i \hat{x}_{2} / 2}-1\right) e^{-i \hat{x}_{1}}= \\
& =i \frac{\left(e^{-i \hat{x}_{2}}-e^{-i \hat{x}_{2} / 2}\right) e^{-i \hat{x}_{1}}}{\hat{x}_{2}}
\end{aligned}
$$

and similar for other $\phi_{t}^{j}, j=5 \ldots 8$.

The equation system in Fourier space is:

$$
\sum_{i} H_{u}^{j i} t^{i}=-F_{u}^{j},
$$

where:

$$
\begin{aligned}
& H_{u}^{j i}=\frac{1}{(2 \pi)^{n}}\left\langle\hat{\phi}_{t}^{j}(-\hat{x}), \hat{\phi}_{t}^{i}(\hat{x}) \hat{U}(\hat{x})\right\rangle, \\
& F_{u}^{j}=\frac{1}{(2 \pi)^{n}}\left\langle\hat{\phi}_{t}^{j}(-\hat{x}), \hat{f}(\hat{x}) \hat{U}(\hat{x})\right\rangle
\end{aligned}
$$

In our case:

$$
\begin{aligned}
& \sum_{i}^{8} t^{i}\left\langle\hat{\phi}_{t}^{j}(-\hat{x}), \hat{\phi}_{t}^{i} \hat{U}\right\rangle=-\left\langle\hat{\phi}_{t}^{j}(-\hat{x}), \hat{f}_{\chi} \hat{U}\right\rangle, \\
& \text { for } j=1, \ldots, 8
\end{aligned}
$$

and for example (for $f_{0}=1$ ):

$$
\begin{gathered}
H^{12}=\frac{1}{(2 \pi)^{2}}\left\langle\hat{\phi}_{t}^{1}(-\hat{x}), \hat{\phi}_{t}^{2}(\hat{x}) \hat{U}(\hat{x})\right\rangle \\
=\frac{1}{(2 \pi)^{2}} \int_{R^{2}} \frac{\left[i\left(e^{-i \hat{x}_{1} / 2}-1\right)\right] \cdot\left[i\left(e^{-i \hat{x}_{1}}-e^{-i x_{1} / 2}\right)\right]}{-\hat{x}_{1} \hat{x}_{1}\left(-\hat{x}_{1}^{2}-\hat{x}_{2}^{2}\right)} d x_{1} d x_{2} \\
F^{1}=\frac{1}{(2 \pi)^{2}} \int_{R^{2}} \hat{\phi}_{t}^{1}(-\hat{x}) \hat{U}(\hat{x}) d \hat{x} \\
=\frac{-1}{(2 \pi)^{2}} \int_{R^{2}}\left[\frac{\left(e^{+i \hat{x}_{1} / 2}-1\right) \dot{ }}{-\hat{x}_{1}\left(-\hat{x}_{1}^{2}-\hat{x}_{2}^{2}\right)}\right] d \hat{x}_{1} d \hat{x}_{2} .
\end{gathered}
$$

Computer implementation of Fourier BEM requires the same skills as classical BEM [1, 3, 6, 7]. Integration with respect to unknowns in the Fourier approach is equivalent to the integral:

$$
H_{u}^{j i}:=\int_{-\infty}^{\infty} \int_{-\infty}^{\infty} \phi_{t}^{j}(\mathbf{x}) \phi_{t}^{i}(\mathbf{y}) U(\mathbf{x}-\mathbf{y}) d x d y,
$$

where:

$i, j$ - number of elements,

$\mathbf{x}=\left[x_{1}, x_{2}\right], \mathbf{y}=\left[y_{1}, y_{2}\right]$ (two dimensional case)

\section{The integral determination}

After dividing the infinite area into four subareas (Fig. 3) we have:

$$
\begin{aligned}
& \int_{-\infty}^{\infty} \int_{-\infty}^{\infty} f(x, y) d x d y=\int_{0}^{\infty} \int_{0}^{\infty} f(x, y) d x d y+\int_{0}^{\infty} \int_{-\infty}^{0} f(x, y) d x d y+ \\
& +\int_{-\infty}^{0} \int_{-\infty}^{0} f(x, y) d x d y+\int_{-\infty}^{\infty} \int_{0}^{\infty} f(x, y) d x d y=\int_{0}^{\infty} \int_{0}^{\infty} f(x, y) d x d y+ \\
& +\int_{0}^{\infty} \int_{0}^{\infty} f(-x, y) d x d y+\int_{0}^{\infty} \int_{0}^{\infty} f(-x,-y) d x d y+\int_{0}^{\infty} \int_{0}^{\infty} f(x,-y) d x d y .
\end{aligned}
$$

To calculate the integrals (46) the Gaussian quadrature with the -1 and 1 integration limits was used. To do that every subarea was transformed to a local coordinate system using the transformations $\mathbf{T}_{1}, \mathbf{T}_{2}$ and $\mathbf{T}_{3}$ (Fig. 4). 


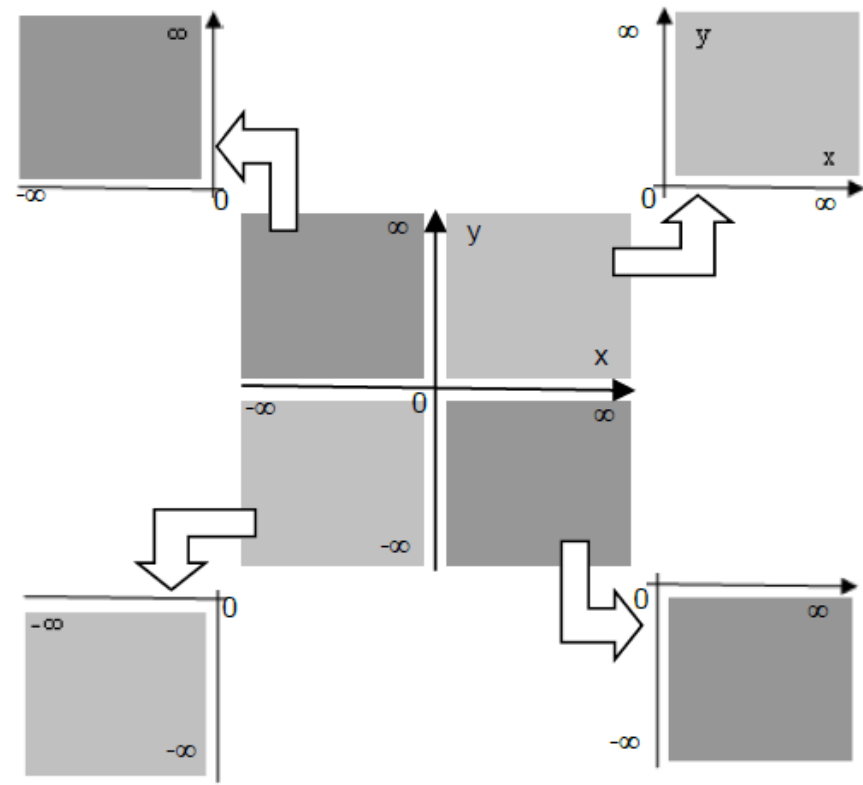

Fig. 3. Dividing the infinite area into four subareas

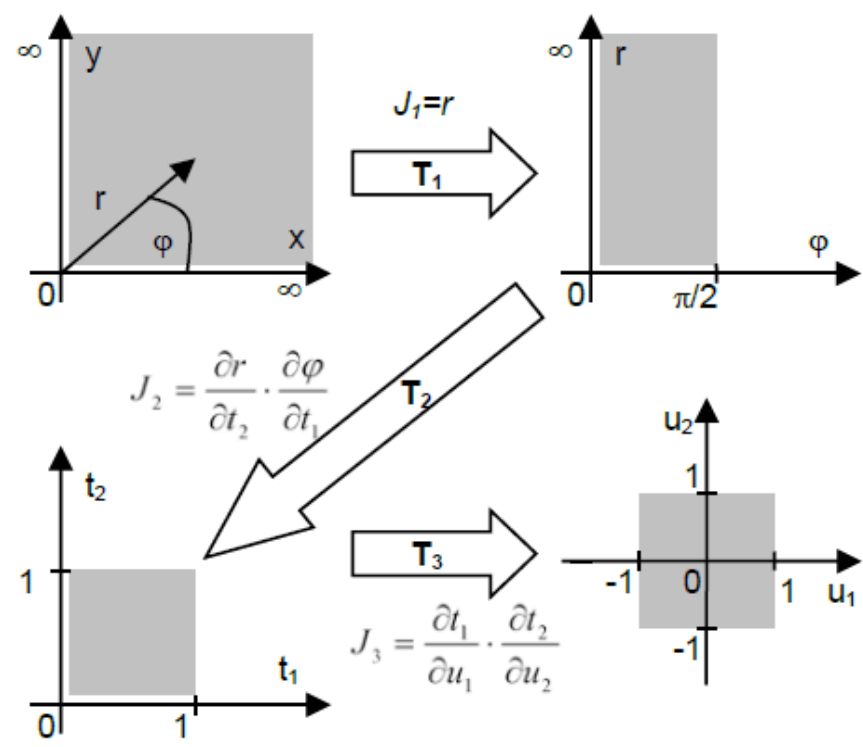

Fig. 4. Transformations to the local coordinate system

\section{Results}

For 8 elements the matrix $\mathbf{H}$ coefficients were calculated symbolically [4] and numerically. Table 1 presents the value of the final solution (the solutions for 8 elements discretization are the same). For the numerical calculation the 80 integration points were used [5].

Table 1. Results

\begin{tabular}{|c|c|c|}
\hline Exact solution & Numerical solution & Relative error \\
\hline 0.256360289033495 & 0.259308496598225 & $1.13 \%$ \\
\hline
\end{tabular}

The numerical calculation of the integrals (46) is very complicated. The calculation based on the proposed algorithm allows us to achieve the $1.13 \%$ accuracy.

\section{Conclusion}

Numerical method for determination of improper integrals occurring in FBEM allowed to determine the results with an error of $1.13 \%$. The Fourier BEM method is more difficult than the standard BEM method but is specially of interest for cases where the fundamental solution is not known.

\section{Bibliography}

[1] Beer G., Programming the boundary element method, John Wiley \& Sons, Ltd, 2001.

[2] Duddeck F., "Generalization of Boundary Element Methods by Fourier Transformation", Springer-Verlag Berlin Heidelberg 2002.

[3] Jabłoński P., Metoda elementów brzegowych w analizie pola elektromagnetycznego, Wydawnictwo Politechniki Częstochowskiej, 2003.

[4] Łukasik E., Pańczyk B., Sikora J, Methods of Optimisation and Data Analysis. Selected Issues, scientific editors: Kesra Nermend and Tomasz Komorowski, chapter 8: Matlab symbolic integration for Galerkin BEM, str. 137-155, Szczecin 2010.

[5] Pańczyk B., Sikora J., Numerical Integration for symmetric Galerkin Boundary Element Method, Przeglaad Elektrotechniczny, No/Vol: 12a/2011, p.36-39.

[6] Sikora Jan, Podstawy metody elementów brzegowych, wydawnictwo Instytutu Elektrotechniki, Warszawa 2009.

[7] Sutradhar A., Paulino G.H., Gray L. J., Symmetric Galerkin Boundary Element Method, Springer-Verlag, Berlin Heidelberg 2008.

[8] Zagórski A., Metody matematyczne fizyki, Oficyna Wydawnicza Politechnik Warszawskiej, Warszawa 2007

\section{Ph.D. Edyta Lukasik \\ e-mail: e.lukasik@pollub.pl}

Edyta Łukasik graduated from mathematics at the Maria Curie-Sklodowska University in Lublin. In 2007 she received $\mathrm{PhD}$ titled "Iterative methods for nonlinear regular singular equations" in Faculty of Mathematics, Physics and Computer Science at UMCS in Lublin. She worked as assistant at the Institute of Computer Science, Lublin University of Technology from 1998 to 2007. Since 2007 she works as adjunct at the Institute of Computer Science, Lublin University of Technology. Research activities: object oriented programming, algorithms and data structures, applications of numerical methods in engineering.

\section{Ph.D. Beata Pańczyk}

e-mail: b.panczyk@pollub.pl

She graduated from mathematics at the Maria CurieSkłodowska University in Lublin. Since 1989 she has been working at the Lublin University of Technology (LTU), where she completed a $\mathrm{PhD}$ in 1996. The doctoral thesis title was Construction of the physical properties distribution image using Impedance Computer Tomography. Currently she is the senior lecturer at the Institute of Computer Science of the LTU. Her didactic and research interest are: object oriented programming, web application development and application of numerical methods in engineering.

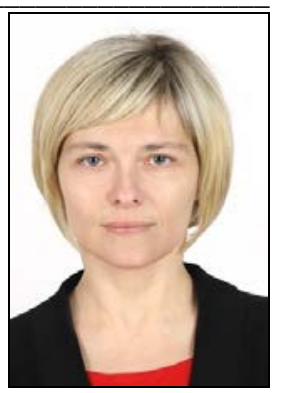

PhD. DSc. Eng. Jan Sikora

e-mail: sik59@wp.pl

PhD. DSc. Eng. Jan Sikora graduated from Warsaw University of Technology Faculty of Electrical Engineering. During 40 years of professional work he has proceeded all grades, including the position of full professor at his alma mater. Since 1998 he has worked for the Institute of Electrical Engineering in Warsaw. In 2008 he has joined Electrical Engineering and Computer Science Faculty In Lublin University of Technology. During 2001-2004 he has worked as a Senior Research Fellow at University College London in the prof. S. Arridge's Group of Optical Tomography. His research interests are focused on numerical methods for electromagnetic field.
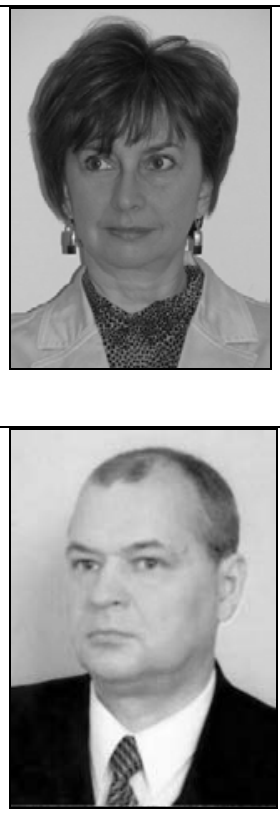